\title{
Antibiotic Prophylaxis for Patients with a History of Total Joint Replacement
}

\author{
Steven F. DeFroda, MD, ME, Eliza Lamin, MD, Joseph A. Gil, MD, Kunal Sindhu, BS, \\ and Scott Ritterman, MD
}

As the population ages and people remain active into the 7 th and 8th decades of life, the frequency of total joint replacement is increasing. Following joint replacement surgery, patients inevitably require various invasive procedures as part of their routine health maintenance, including, but not limited to, dental care, colonoscopy, cystoscopy, and cardiac catheterization. There is scant evidence to support the use of periprocedural prophylactic antibiotics in the prevention of late total joint infection. The guidelines for the usage of periprocedural antibiotics have changed as knowledge of the pathophysiology of joint infection has evolved. We review the current recommendations from subspecialty academic organizations regarding antibiotic prophylaxis for patients undergoing routine urologic, gastrointestinal, dental, and cardiac procedures after total joint replacement. (J Am Board Fam Med 2016;29:500-507.)

Keywords: Antibacterial Agents, Antibiotic Prophylaxis, Infectious Arthritis, Replacement Arthroplasty

It is estimated that 2.5 million Americans are living with total hip replacements, while 4.7 million currently have total knee replacements. ${ }^{1}$ These numbers are expected to increase as patients live longer and remain active into the sixth, seventh, and eighth decades of life. As the number of patients with total joint replacement increases, so too does the need for other care providers to be aware of this population of patients and the unique challenges they present in other areas of their care. One area that has been the subject of much controversy is the need for antibiotic prophylaxis for patients with total joint replacements who are undergoing outpatient urologic, gastrointestinal, dental, or cardiac interventions. With the number of total joint

This article was externally peer reviewed.

Submitted 16 December 2015; revised 30 March 2016; accepted 11 April 2016.

From the Department of Orthopaedics, Alpert Medical School, Brown University, Providence, RI (SFD, JAG, SR); the Department of Urology, University of Pennsylvania, Perelman Center for Advanced Medicine, Philadelphia (EL); and Department of Orthopaedics Alpert Medical School of Brown University, 593 Eddy Street, Providence, RI 02903 (KS).

Funding: none.

Conflict of interest: none declared.

Corresponding author: Steven F. DeFroda, MD, ME, Department of Orthopaedics, Alpert Medical School, Brown University, 593 Eddy Street, Providence, RI 02903 (E-mail: sdefroda@gmail.com). replacements increasing, prosthetic joint infection will become a larger burden on our health care system. Transient bacteremia, which leads to the seeding of a prosthetic joint and resultant joint infection, is a hypothesized mechanism of infection during various interventions; however, this has never been definitively proven. ${ }^{2}$ Southwood et $\mathrm{al}^{3}$ showed that with a high bacterial load, bacteremia could lead to a prosthetic infection up to 3 weeks after joint replacement in a rabbit population; however, the rabbits became resistant to infection 3 weeks postoperatively. A study of 1000 patients with 1112 joint replacements advised patients to not take prophylactic antibiotics before any dental or surgical procedures. In this population, 284 infections developed, but no hematogenous joint infections were reported; only 3 patients developed bacteremia ( 2 had rheumatoid arthritis). ${ }^{4}$ On the contrary, other authors argue that it has been proven that dental and surgical procedures are capable of causing transient bacteremia. ${ }^{5,6}$ In the gastrointestinal tract, transient bacteremia infiltrating via bacterial translocation across the intestinal mucosa has occurred in patients with immune compromise and malignancy; however, it is uncommon in healthy individuals. ${ }^{7}$

While it is standard practice to give perioperative antibiotics to patients having joint replacement 
surgery, there is much controversy regarding the need for routine prophylaxis in patients with a history of joint replacement undergoing various outpatient procedures. As a part of routine health care maintenance, patients require a multitude of outpatient procedures performed by numerous subspecialty providers, including but not limited to urologists, gastroenterologists, dentists, and cardiologists. Patients with joint replacement may question both these providers and their primary care provider about the need for any special antibiotic requirements, and they often receive contradictory recommendations.

In 2009 the American Academy of Orthopaedic Surgeons (AAOS) put out a blanket statement with regards to antibiotic prophylaxis. They recommended "consideration" of antibiotics for all patients with total joint replacement who were undergoing any procedure with the potential to cause bacteremia and identified a high-risk group of patients, including immunocompromised patients; those with inflammatory arthropathy; immunosuppressed patients; patients with HIV; those with previous joint infection, hemophilia, type 1 diabetes, or malignancy; and patients with a megaprosthesis. ${ }^{8}$ They do not recommend antibiotic prophylaxis for patients with extrasynovial implants (eg, plates, screws). However, these generic recommendations vary for different subspecialty organizations such as the American Urologic Association (AUA), American Heart Association (AHA), and American Dental Association (ADA).

Being aware of the evidence associated with prosthetic infection during these procedures as well as the guidelines within each of these academic organizations is important in optimizing patient care and limiting both the rate of infection and the adverse effects of antibiotic therapy. A literature review was completed by performing a PubMed search for relevant literature on prophylactic antibiotics following joint replacement surgery using the terms urologic procedures joint infections, antibiotic prophylaxis after joint surgery dental procedures, GI procedures joint infection, cardiac procedures prosthetic joint infections, and dental procedures prosthetic joint infections. These terms resulted in 240 publications, 215 of which were either irrelevant or duplicates. Only manuscripts written in English were considered. Relevant articles that were referenced within the included manuscripts were also reviewed, as were references from the national subspecialty organization guidelines.

\section{Use of Antibiotics \\ Urologic Procedures}

Transient bacteremia is a common consequence of urologic surgery. Transient bacteremia after urologic surgery occurs in $6.1 \%$ of patients without antibiotics and $2.1 \%$ with antibiotics. ${ }^{9}$ Intraoperative manipulation of the genitourinary $(\mathrm{GU})$ tract can allow small amounts of bacteria to be transferred into the bloodstream during routine bleeding during surgery. While there is a theoretical risk as well as a logical connection between bacteremia, seeding, and prosthetic joint infection, there are no definitive data that provide a causal link between urologic procedures and prosthetic joint infections. ${ }^{10}$ The risk of bacteremia depends on several factors including preoperative urinary tract infection, invasiveness of the procedure, and use of prophylactic antibiotics. However, there is such a paucity of data on the incidence of periprosthetic infection following urologic procedures that the Canadian Urologic Association does not provide in their antibiotic guidelines any information on patients with joint replacements. ${ }^{11}$ In addition, the European Urology Association does not mention special precautions to be taken in patients with a joint replacement. ${ }^{12}$

In one case-control study there was no difference in the rate of periprosthetic joint infection between those who had a GU procedure and those who did not. ${ }^{13}$ The study compared 339 patients admitted with a periprosthetic joint infection with 339 patients who were admitted at the same time without a joint infection. The rate of GU procedures (about 15\%) was similar in the infected group and the noninfected group. Results were similar in patients whose joints were 6 months, 1 year, and $>1$ year old.

The AAOS does not provide specific recommendations for urologic procedures; however, the AUA provides urologists with a best-practice statement regarding the use of antibiotic prophylaxis in patients with joint replacements. According to the AUA, patients who meet 1 criterion from 1 or both of the following categories should be prescribed prophylactic antibiotics. The categories are divided into patients who have an increased risk of hematogenous total joint infection and patients under- 
going procedures that have a higher rate of causing bacteremia. The first category includes patients within 2 years of their joint replacement, immunocompromised patients, and patients with the at least one of the following comorbidities: previous joint infections, malnourishment, hemophilia, HIV infection, diabetes, and malignancy. The second category includes any patient undergoing kidney stone manipulation, upper urinary tract manipulation (ureteroscopy, percutaneous nephrolithotomy, extracorporeal shock wave lithotripsy), transrectal prostate biopsy, or bowel manipulation, and those who have a higher risk of colonization because of an indwelling catheter, clean intermittent catheterization, urinary retention, recent urinary tract infection, an indwelling ureteral stent, or urinary diversion. ${ }^{14}$ The prophylactic antibiotic recommendation for patients who meet the criteria is a single dose of a fluoroquinolone given orally 1 to 2 hours preoperatively. Alternatively, a combination of ampicillin (or vancomycin if the patient is allergic to penicillin) and gentamicin can be given 30 to 60 minutes preoperatively. ${ }^{14}$ The goal of prophylactic antibiotics is to prevent hematogenous seeding of joint replacements. Joint replacements are significantly more likely to be infected in the first 2 years after implantation, which is likely the reason the AUA recommends antibiotics for patients who had a joint replacement $<2$ years before their procedure. $^{15}$

Based on these guidelines and the current literature, it can be said that not all patients with a joint replacement need antibiotics; however, it is important to go through a patient's history to determine whether they have the potential for a high rate of hematogenous spread or whether the procedure they are undergoing has a higher rate of causing bacteremia. Patients meeting both of these criteria should receive prophylactic antibiotics. As more literature becomes available, it may show that not even all these patients should be receiving antibiotics, but in the absence of large clinical trials it is important to continue with the current AUA guidelines.

\section{Gastrointestinal Procedures}

Bacteremia following gastrointestinal endoscopic procedures is not uncommon. The incidence of bacteremia after a standard colonoscopy, for example, has been estimated to be between $0 \%$ and $5 \% .{ }^{16}$ Even in those gastrointestinal procedures deemed to be "high risk" for bacteremia, such as dilation of esophageal strictures, endoscopic sclerotherapy of varices, and endoscopic retrograde cholangiopancreatography, the incidence of bacteremia has still been found to be lower than $22 \% .^{17,18}$ By contrast, common activities like brushing one's teeth and flossing are associated with an incidence of bacteremia between $20 \%$ and $68 \% .^{18}$

Previous investigations have revealed a few instances of prosthetic joint infections following gastrointestinal endoscopic procedures. ${ }^{4,19}$ In 2008, Banerjee et $\mathrm{al}^{18}$ reported that there were 2 case reports of pyogenic arthritis in patients with orthopaedic prostheses. A 2013 case-control study examining prosthetic joint infections found that $17 \%$ of such infections were caused by bacteria native to the gastrointestinal tract. ${ }^{19}$ The same study found that esophagogastroduodenoscopy with biopsy within 2 years of primary arthroplasty was associated with a significantly increased risk of prosthetic joint infection. However, this was the first study to demonstrate this association, and there are few data on the incidence of this relationship. ${ }^{19}$ Given the low incidence of joint infections following endoscopy, prophylactic antibiotics are not currently recommended by the American Society of Gastroenterologists or the American Society of Colon and Rectal Surgeons. ${ }^{16,20}$ In addition, prophylactic antibiotics are also no longer recommended in the antibiotic prophylaxis guidelines from the AAOS. ${ }^{2}$

\section{Dental Procedures}

It has been estimated that $6 \%$ to $13 \%$ of prosthetic joint infections result from organisms that originate in the oral cavity. ${ }^{21}$ Given the considerable costs, morbidity, and mortality associated with these infections, and the fact that dental procedures are known to induce transient bacteremia, ${ }^{16,22}$ the use of prophylactic antibiotics by orthopedists before dental procedures became widespread in the 1970s. ${ }^{23}$ However, the data supporting this practice proved to be limited. ${ }^{24}$ One study by Coulter et $\mathrm{al}^{5}$ showed a reduction in the incidence of bacteremia with the use of antibiotics-from $63 \%$ to $35 \%$; however, there was no proof of a significant difference in infection rate in either group. In 1997, in an attempt to provide clinicians with better guidance regarding the prophylactic use of antibiotics in the setting of dental procedures, the $\mathrm{ADA}$ and the AAOS issued joint guidelines that stated that "an- 
tibiotic prophylaxis is not indicated for dental patients with pins, plates and screws, nor is it routinely indicated for most dental patients with total joint replacements." Instead, prophylaxis was only recommended for a small group of individuals at high risk of infection. ${ }^{25}$ The guidelines were revised in 2003, but they largely remained consistent with the 1997 recommendations. ${ }^{26}$

In 2009, however, without the endorsement of the $\mathrm{ADA}$, the AAOS unilaterally issued new recommendations, arguing that the significant morbidity and mortality associated with prosthetic joint infections justified clinicians to "consider antibiotic prophylaxis for all total joint replacement patients before any invasive procedure that can cause bacteremia." This statement, however, did not meet the AAOS criteria for evidence-based guidelines. ${ }^{27}$ The ADA responded by pointing out that there was inadequate evidence to justify the change from the 2003 guidelines. ${ }^{26}$ This statement issued by the ADA has since been supported by numerous studies that have questioned the use of prophylactic antibiotics in patients with prosthetic joints. A large case-control study by Berbari et $\mathrm{al}^{22}$ showed that antibiotic prophylaxis before dental procedures was not associated with a decreased risk of prosthetic joint infections and suggested that the 2009 guidelines should be reconsidered. Studies by Matar et $\mathrm{al}^{28}$ and Skaar et $\mathrm{al}^{29}$ came to similar conclusions.

In 2012 the AAOS, once again in conjunction with the ADA, reversed itself, stating that clinicians "might consider discontinuing the practice of routinely prescribing prophylactic antibiotics for patients with hip and knee prosthetic joint implants undergoing dental procedures," and the AAOS suggested that patient preference should play a significant role in determining the ultimate course of action. $^{30}$ In 2014 the ADA convened an expert panel to evaluate its 2012 recommendations; this panel recommended that prophylactic antibiotics should not be given before dental procedures in patients with prosthetic joints. ${ }^{31}$ Despite these recommendations, Colterjohn et $\mathrm{al}^{32}$ showed that orthopedic surgeons continue to be far more likely than oral surgeons to prescribe prophylactic antibiotics before dental procedures.

Interestingly, the AHA does not share the same view as the AAOS with regard to prophylaxis for patients with implantable heart devices. The AHA stance is that because of the high prevalence of staphylococcal infection (which is not native to the mouth) in cardiovascular implantable devices, there is no role for antibiotic prophylaxis during dental procedures for patients with this device. ${ }^{33}$ This is interesting to note because Staphylococcus aureus and Staphylococcus epidermidis have been shown to be the most common infecting organisms in prosthetic joint infection. While comparing infections of cardiac devices to total joint replacements following dental procedures may be inappropriate, it may serve as a good area of investigation for the AAOS going forward in making potentially improved recommendations regarding prophylaxis.

\section{Cardiac Procedures}

Cardiovascular implantable electronic devices (CIEDs), which include permanent pacemakers and implantable cardioverter-defibrillators, have become essential to the management of cardiovascular disease in the United States. ${ }^{33,34}$ Between 1999 and 2003, the number of new CIED implantations increased by $49 \%$, driven primarily by new implantable cardioverter-defibrillators. ${ }^{35}$ Patients receiving CIEDs tend to be older and suffer from more comorbidities than the population as a whole. $^{36}$ The increased prevalence of CIEDs, coupled with their presence in older, sicker patients, has led to an increased incidence of CIED infection. $^{33,34}$ Notably, the number of hospitalizations resulting from CIED infections increased 3.1-fold between 1996 and 2003, significantly outstripping the increase in CIED prevalence. ${ }^{35}$

Endocarditis and device-related infections are the most common types of infections complicating CIED placement. ${ }^{33,34}$ Between $60 \%$ to $80 \%$ of these infections are caused by Staphylococcus species; $42 \%$ are caused by coagulase-negative Staphylococcus alone. ${ }^{34,37}$ CIED infections tend to be characterized by high rates of morbidity and mortality. ${ }^{34,35}$ A 2009 study, for example, found an 18\% all-cause mortality from CIED infections at 6 months. ${ }^{38}$ Given the high risks associated with these infections, the AHA recommends the use of prophylactic antibiotics in all patients, regardless of prosthetic joint status, before CIED placement. Specifically, they recommend that cefazolin be administered intravenously within 1 hour before the incision or vancomycin within 2 hours of the incision. ${ }^{34}$ Because of a lack of evidence regarding their efficacy and concerns about their risks and costs, however, postoperative antibiotics and prophylactic antibiotics before other invasive procedures, in- 
cluding dental procedures, not related to CIED manipulation are not recommended. ${ }^{33,34}$ While there have not been many studies looking at the use of antibiotics in patients with both prosthetic joints and CIED implants, we believe that it is prudent to follow the AHA guidelines regarding the use of antibiotics before CIED placement for all patients, including those with prior arthroplasty, until further studies clarify this issue. In addition, the AHA does not recommend antibiotics for routine procedures in which antibiotics are not routinely given for those without joint replacement.

\section{Discussion}

Patients with history of joint replacement often ask their physicians questions regarding the need for prophylactic antibiotics before undergoing an invasive outpatient procedure such as dental work or a urologic procedure. Different subspecialty academic organizations and regional practice patterns may influence the decision to prescribe prophylactic antibiotics. Ultimately the decision should be based on the risk of infection as well as the morbidity associated with periprosthetic joint infection. Many orthopedists argue that prosthetic joint infection is a devastating complication of joint arthroplasty and should be avoided at all costs. However, primary care physicians argue that antibiotic administration is not without consequence, potentially causing the emergence of drug-resistant organisms, mild drug-related adverse effects such as swelling or itching, and even more severe adverse effects such as Clostridium difficile colitis. While urologic, gastrointestinal, dental, and cardiac procedures have all been proven to induce bacteremia, daily activity such as teeth brushing also results in bacteremia, and prosthetic joint infection via hematogenous seeding has never been definitively proven in humans. ${ }^{2}$ In addition, in today's current health care landscape it is important to consider the cost of delivering health care as well as the cost of a devastating complication such as prosthetic infection. Slover et $\mathrm{al}^{39}$ analyzed the cost associated with prosthetic infection compared with prophylactic antibiotic usage. They used $\beta$-lactams as a model antibiotic for prophylaxis and, using the incidence of various adverse effects such as anaphylaxis $(0.015-0.004 \%),{ }^{40}$ C. difficile infection $(0.0000067 \%),{ }^{41}$ and rash $(5.1 \%),{ }^{42}$ they estimated the average cost of antibiotic complication per prescription to be
$\$ 14.30 .^{39}$ Based on the available data, the average cost of a prosthetic joint infection was determined to be $\$ 90,000$. A cost-to-benefit analysis demonstrated that if the risk of infection was $<0.75 \%$, prophylactic antibiotics were not cost-effective; however, they were demonstrated to be cost-effective if the risk was $1.2 \%$. If the risk of prosthetic joint injection was $2.1 \%$, antibiotics would only need to reduce risk by $25 \%$ to be cost-effective. ${ }^{39}$ While there are no reported rates $>0.75 \%$, from a strictly cost-effectiveness perspective, the results of this study determined that antibiotics are only costeffective in certain patients who are at a high risk of acquiring a prosthetic joint infection, including those with immune compromise, recent joint replacement (within 2 years), diabetes, or inflammatory arthritis. ${ }^{43}$

Overall, the recommendation regarding the utilization of prophylactic antibiotics in patients with joint replacement in the setting of invasive procedures varies depending on the type of provider making the recommendation. A survey study of orthopedist surgeons, urologists, and dentists assessed each group's thoughts on antibiotic prophylaxis. All 3 groups thought that patients should "definitely" inform their physician of their joint replacement before a procedure. Urologists felt that patients with an existing joint replacement "probably" needed antibiotics before a routine or prolonged procedure, whereas dentists replied "probably not" and "unsure." Interestingly, orthopedist surgeons felt that their patients "definitely" needed antibiotics before urologic manipulation and "probably" required it before dental treatment, regardless of procedure length. ${ }^{44}$ This study reveals that patients will get inconsistent recommendations depending on the type of provider they ask. It also demonstrates the importance of communication regarding the various subspecialty organizations and the need for collaborative research going forward to best examine the risks of infection and its prevention. Ultimately, our review shows that the literature suggests recommending prophylactic antibiotics only for patients with total joint replacement in the event that they are undergoing a major urologic procedure (as previously described) or undergoing a routine urologic or dental procedure with 1 or more of the following risk factors: immunocompromise, previous joint infections, malnourishment, hemophilia, HIV, diabetes, malignancy, or a joint implanted within the past 2 
Table 1. Summary of Antibiotic Prophylaxis Recommendations Re, $, 14,16,20,30,31,34^{2}$

\begin{tabular}{|c|c|c|c|}
\hline $\begin{array}{l}\text { Type of } \\
\text { Procedure }\end{array}$ & Recommending Organization & Antibiotics Recommended? & Regiment \\
\hline Urologic & American Urologic Association & $\begin{array}{l}\text { Yes, in high-risk patients or } \\
\text { procedures }{ }^{16 *}\end{array}$ & $\begin{array}{l}\text { Fluoroquinolone PO } 1-2 \text { hours } \\
\text { preoperatively OR ampicillin }+ \\
\text { gentamicin 30-60 minutes } \\
\text { preoperatively }\end{array}$ \\
\hline Gastrointestinal & $\begin{array}{l}\text { American Society of } \\
\text { Gastroenterologists/American } \\
\text { Society of Colon and Rectal } \\
\text { Surgeons }\end{array}$ & No & N/A \\
\hline \multirow[t]{2}{*}{ Dental } & $\begin{array}{l}\text { American Academy of Orthopaedic } \\
\text { Surgeons }\end{array}$ & $\mathrm{No}^{+}$ & Cephalexin 2 g PO 1 hour before \\
\hline & American Dental Association & $\mathrm{No}^{9,26,31}$ & N/A \\
\hline Cardiac & American Heart Association & $\begin{array}{l}\text { Preoperatively for all CIEDs; } \\
\text { not recommended before } \\
\text { other invasive procedures } \\
\text { or postoperatively }\end{array}$ & $\begin{array}{l}\text { Cefazolin } 1 \text { hour preoperatively } \\
\text { or vancomycin } 2 \text { hours } \\
\text { preoperatively }\end{array}$ \\
\hline
\end{tabular}

*Defined by the American Urologic Association as patients within 2 years of their joint replacement; immunocompromised patients; or patients with previous joint infections, malnourishment, hemophilia, HIV infection, diabetes, or malignancy. High-risk procedures include kidney stone manipulation, ureteroscopy, percutaneous nephrolithotomy, extracorporeal shock wave lithotripsy, transrectal prostate biopsy, and bowel manipulation. Other high risks include patients with indwelling catheters, clean intermittent catheterization, urinary retention, recent urinary tract infection, indwelling ureteral stent, or urinary diversion.

${ }^{\dagger}$ Should be addressed on a patient-by-patient basis and incorporate both patient and physician preference as well as the presence of risk factors for infection, such as immunocompromised patients; those with inflammatory arthropathy; immunosuppressed patients; patients with HIV; those with previous joint infection, hemophilia, type 1 diabetes, or malignancy; and patients with a megaprostheses. CIED, cardiac implantable electronic device; N/A, not applicable; PO, by mouth.

years. Patients undergoing routine cardiac procedure should receive any antibiotics routinely given for the procedure but do not need additional antibiotic prophylaxis; those undergoing gastrointestinal procedures do not require prophylactic antibiotics (Table 1).

\section{Conclusion}

The decision to use prophylactic antibiotics in patients with joint replacement in the setting of invasive procedures is one that is shared between orthopedist surgeons, primary care physicians, and subspecialists performing the invasive procedures. Patients may get varying opinions depending on which provider they ask as a result of differing recommendations within each subspecialty's governing body. Based on the evidence, healthy individuals with a $>2$ year history of uncomplicated joint arthroplasty should not typically receive antibiotic prophylaxis. Patients should be informed of the current guidelines and be encouraged to make an informed decision based on the available information. While it may be tempting for orthopedic surgeons to recommend antibiotics as a preventive measure, the administration of antibiotics is not benign and may not be cost-effective unless it is used in a population that has a high risk for infection. By being aware of the latest recommendations and the literature regarding the risks of prosthetic joint infection, providers can optimize the perioperative protocol associated with their respective interventions while reducing the costs and morbidity affiliated with improper antibiotic usage as well as the incidence of prosthetic joint infection.

\section{References}

1. 2.5 million Americans living with an artificial hip, 4.7 million with an artificial knee [press release]. AAOS Newsroom, March 14, 2014. Available from: http:// newsroom.aaos.org/media-resources/Press-releases/ 25-million-americans-living-with-an-artificial-hip-47million-with-an-artificial-knee.htm. Accessed March 20, 2015.

2. Watters W, Rethman MP, Hanson NB, et al. Prevention of orthopaedic implant infection in patients undergoing dental procedures. J Am Acad Orthop Surg 2013;21:180-9.

3. Southwood RT, Rice JL, McDonald PJ, Hakendorf PH, Rozenbilds MA. Infection in experimental arthroplasties. Clin Orthop Relat Res 1987;224: 33-6.

4. Ainscow DA, Denham RA. The risk of haematogenous infection in total joint replacements. J Bone Joint Surg Br 1984;66:580-2. 
5. Coulter WA, Coffey A, Saunders ID, Emmerson AM. Bacteremia in children following dental extraction. J Dent Res 1990;69:1691-5.

6. Lockhart PB. An analysis of bacteremias during dental extractions. A double-blind, placebo-controlled study of chlorhexidine. Arch Intern Med 1996;156: 513-20.

7. Srivastava A, Walter N, Atkinson P. Streptococcus bovis infection of total hip arthroplasty in association with carcinoma of colon. J Surg Orthop Adv 2010; 19:125-8.

8. Antibiotic prophylaxis for patients after total joint replacement. Information statement from the American Academy of Orthopaedic Surgeons. Available from: http://orthodoc.aaos.org/davidgrimmmd/Antibiotic Prophylaxis for Patients after Total Joint Replacement. pdf. Accessed March 26, 2016.

9. Alsaywid BS, Smith GH. Antibiotic prophylaxis for transurethral urological surgeries: systematic review. Urol Ann 2013;5:61-74.

10. Mazur DJ, Fuchs DJ, Abicht TO, Peabody TD. Update on antibiotic prophylaxis for genitourinary procedures in patients with artificial joint replacement and artificial heart valves. Urol Clin North Am 2015;42:441-7.

11. Mrkobrada M, Ying I, Mokrycke S, et al. CUA guidelines on antibiotic prophylaxis for urologic procedures. Can Urol Assoc J 2015;9:13-22.

12. Grabe M, Bartoletti R, Bjerklund-Johansen TE, et al. UTIs in children. In: Guidelines on urological infections. Arnhem, the Netherlands: European Association of Urology; 2015: 33-40. Available from: http://uroweb.org/wpcontent/uploads/19-Urological-infections_LR2.pdf. Accessed May 18, 2016.

13. Gupta A, Osmon D, Hanssen A, et al. Genitourinary procedures as risk factors for prosthetic hip or knee infection: a hospital-based prospective case-control study. Open Forum Infect Dis 2015;2:ofv097.

14. Wolf JS, Bennett CJ, Dmochowski RR, Hollenbeck BK, Pearle MS, Schaeffer AJ. Best practice policy statement on urologic surgery antimicrobial prophylaxis. J Urol 2008;179:1379-90.

15. Phillips JE, Crane TP, Noy M, Elliott TSJ, Grimer RJ. The incidence of deep prosthetic infections in a specialist orthopaedic hospital: a 15-year prospective survey. J Bone Joint Surg Br 2006;88:943-8.

16. Oliver G, Lowry A, Vernava A, et al. Practice parameters for antibiotic prophylaxis-supporting documentation. The Standards Task Force. The American Society of Colon and Rectal Surgeons. Dis Colon Rectum 2000;43:1194-200.

17. Nelson DB, Sanderson SJ, Azar MM. Bacteremia with esophageal dilation. Gastrointest Endosc 1998; 48:563-7.

18. Banerjee S, Shen B, Baron TH, et al. Antibiotic prophylaxis for GI endoscopy. Gastrointest Endosc 2008;67:791-8.
19. Coelho-Prabhu N, Oxentenko AS, Osmon DR, et al. Increased risk of prosthetic joint infection associated with esophago-gastro-duodenoscopy with biopsy. Acta Orthop 2013;84:82-6.

20. Khashab MA, Chithadi KV, Acosta RD, et al. Antibiotic prophylaxis for GI endoscopy. Gastrointest Endosc 2015;81:81-9.

21. Young H, Hirsh J, Hammerberg EM, Price CS. Dental disease and periprosthetic joint infection. J Bone Joint Surg Am 2014;96:162-8.

22. Berbari EF, Osmon DR, Carr A, et al. Dental procedures as risk factors for prosthetic hip or knee infection: a hospital-based prospective case-control study. Clin Infect Dis 2010;50:8-16.

23. Lattimer GL, Keblish PA, Dickson TBJ, Vernick CG, Finnegan WJ. Hematogenous infection in total joint replacement. Recommendations for prophylactic antibiotics. JAMA 1979;242:2213-4.

24. Management of dental patients with prosthetic joints. Council on Dental Therapeutics. J Am Dent Assoc 1990;121:537-8.

25. Antibiotic prophylaxis for dental patients with total joint replacements. American Dental Association; American Academy of Orthopaedic Surgeons. J Kans Dent Assoc 1997;82:14, 16-17.

26. Little JW, Jacobson JJ, Lockhart PB. The dental treatment of patients with joint replacements: a position paper from the American Academy of Oral Medicine. J Am Dent Assoc. 2010;141:66771.

27. Alao U, Pydisetty R, Sandiford NA. Antibiotic prophylaxis during dental procedures in patients with in situ lower limb prosthetic joints. Eur J Orthop Surg Traumatol 2015;25:217-20.

28. Matar WY, Jafari SM, Restrepo C, Austin M, Purtill JJ, Parvizi J. Preventing infection in total joint arthroplasty. J Bone Joint Surg Am 2010;92(Suppl 2): $36-46$.

29. Skaar DD, O'Connor H, Hodges JS, Michalowicz BS. Dental procedures and subsequent prosthetic joint infections: findings from the Medicare Current Beneficiary Survey. J Am Dent Assoc 2011;142: 1343-51.

30. Hamedani Sh. A Clinical practice update on the latest AAOS/ADA guideline (December 2012) on prevention of orthopaedic implant infection in dental patients. J Dent (Shiraz) 2013;14:49-52.

31. Sollecito TP, Abt E, Lockhart PB, et al. The use of prophylactic antibiotics prior to dental procedures in patients with prosthetic joints: evidence-based clinical practice guideline for dental practitioners-a report of the American Dental Association Council on Scientific Affairs. J Am Dent Assoc 2015;146:116.e8.

32. Colterjohn T, de Beer J, Petruccelli D, Zabtia N, Winemaker M. Antibiotic prophylaxis for dental procedures at risk of causing bacteremia among posttotal joint arthroplasty patients: a survey of Canadian 
orthopaedic surgeons and dental surgeons. J Arthroplasty. 2014;29:1091-7.

33. Baddour LM, Epstein AE, Erickson CC, et al. A summary of the update on cardiovascular implantable electronic device infections and their management: a scientific statement from the American Heart Association. J Am Dent Assoc 2011;142:15965.

34. Baddour LM, Epstein AE, Erickson CC, et al. Update on cardiovascular implantable electronic device infections and their management: a scientific statement from the American Heart Association. Circulation 2010;121:458-77.

35. Voigt A, Shalaby A, Saba S. Rising rates of cardiac rhythm management device infections in the United States: 1996 through 2003. J Am Coll Cardiol 2006; 48:590-1.

36. Zhan C, Baine WB, Sedrakyan A, Steiner C. Cardiac device implantation in the United States from 1997 through 2004: a population-based analysis. J Gen Intern Med 2008;23(Suppl 1):13-9.

37. Sohail MR, Uslan DZ, Khan AH, et al. Management and outcome of permanent pacemaker and implantable cardioverter-defibrillator infections. J Am Coll Cardiol 2007;49:1851-9.
38. Baman TS, Gupta SK, Valle JA, Yamada E. Risk factors for mortality in patients with cardiac devicerelated infection. Circ Arrhythm Electrophysiol 2009;2:129-34.

39. Slover JD, Phillips MS, Iorio R, Bosco J. Is routine antibiotic prophylaxis cost effective for total joint replacement patients? J Arthroplasty 2015;30:543-6.

40. Idsoe O, Guthe T, Willcox RR, de Weck AL. Nature and extent of penicillin side-reactions, with particular reference to fatalities from anaphylactic shock. Bull World Health Organ 1968;38:159-88.

41. Hirschhorn LR, Trnka Y, Onderdonk A, Lee ML, Platt R. Epidemiology of community-acquired Clostridium difficile-associated diarrhea. J Infect Dis 1994;169:127-33.

42. Bigby M, Jick S, Jick H, Arndt K. Drug-induced cutaneous reactions. A report from the Boston Collaborative Drug Surveillance Program on 15,438 consecutive inpatients, 1975 to 1982 . JAMA 1986; 256:3358-63.

43. Jevsevar DS. Shared decision making tool: should I take antibiotics before my dental procedure? J Am Acad Orthop Surg 2013;21:190-2.

44. Kingston R, Kiely P, McElwain JP. Antibiotic prophylaxis for dental or urological procedures following hip or knee replacement. J Infect 2002;45:243-5. 